\title{
Transforming Economies and Generating Sustainable "Green" Economic Growth After the COVID-19 Pandemic through General Collective Intelligence
}

Andy E. Williams

Nobeah Foundation, Nairobi, Kenya

Email: awilliams@nobeahfoundation.org

\begin{abstract}
The lockdown of economic activity in many countries as a measure to stop the spread of the COVID19 pandemic has led to high levels of unemployment and other indicators of a potentially upcoming economic crisis. As a gauge of the seriousness of these concerns some have suggested that current levels of some of these indicators have not been seen since the time of the great depression. This paper explores how General Collective Intelligence, a recent innovation in group decision-making systems, might reliably generate the economic growth needed to avert such a crisis where not reliably achievable otherwise. Current group decision-making systems, whether choosing a human decision-maker, consensus voting on decisions, or automated decision-systems such as conventional collective intelligence, have been suggested to lack the capacity to maximize more than a very few group outcomes due to specific limitations. Since impact on collective well-being is determined by impact on an open set of outcomes, this implies lack of the capacity to maximize the range of dimensions of impact on well-being for groups if that range is too broad. If so, the breadth of impact required to achieve sustainable "green" economic development while simultaneously solving hunger, solving the environmental degradation that some have linked to climate change, as well as providing maximal access to healthcare, education, and other resources, may not be reliably possible with current systems. General Collective Intelligence or GCI replicates the adaptive problem solving mechanisms by which nature has demonstrated the ability to optimally respond to an unlimited set of problems, and demonstrated the ability to potentially increase sustainability per unit of resources by orders of magnitude so that life is reliably self-sustaining. This paper explores why GCI can potentially be used to reliably drive self-sustaining economic growth to revive economies in the aftermath of the COVID19 pandemic, and why GCI has the potential to reliably drive a transformation to sustainable green economies while doing so.
\end{abstract}

\section{Introduction}

Recent work suggests that current group decision-making systems may lack the capacity to address entire categories of problems [1], including massive multidimensional optimization of group problems. That is, they may lack the capacity for simultaneously optimizing outcomes for groups according to a wide range of criteria that require massive cooperation, including criteria such as economic benefit, environmental impact, as well as impact on health systems, education systems, energy systems, water and sanitation and other systems, and doing so for potentially thousands, millions, or even billions of people. Global tipping points have been described as points at which terrestrial systems such as ecosystems, elements of the climate system, or elements of human civilizations shift radically and potentially irreversibly into a different state [2], [3], [4]. Lacking this capacity for simultaneous optimization of outcomes for groups, if global tipping points exist, then current group decision-making systems may lack the capacity to reliably avoid those tipping points.

Current decision-making systems may lack the capacity to address entire categories of problems, according to a recently developed framework for modeling cognition [5] due to the lack of sufficient cognitive functionality for solving such general problems. One reason to conclude this is that they lack a common means to measure optimal outcomes across all problems. Another reason is that current decision-making systems lack a stable mechanism to continually adapt to become more fit at their 
decision-making functions across all potential decisions. One might also conclude that current decision-making systems lack the capacity for massively cooperative general problem solving because these two elements of functionality are theorized in the FMF as being required for this cooperation.

This Functional Modeling Framework (FMF) defines a General Collective Intelligence or GCI as a collective intelligence with general problem solving ability. Where Collective Intelligence (CI) uses the intelligence of crowds to optimize outcomes, having general problem solving ability, a GCI must also has the capacity to further optimize outcomes by choosing the optimal problem to solve. A recent developed model of GCI as defined within this FMF (the "change engine") implements the properties suggested by the framework as being required to achieve this general problem solving ability (collective cognition).

General Collective Intelligence or GCI replicates the adaptive problem solving mechanisms by which nature has demonstrated the ability to adapt organisms at every level, whether at the level of any cell, at the level of any group of cells, or at the level of the entire organism, so that the organism as a collective of cells might optimally respond to an unlimited set of problems. In the sense that an overturned petri dish full of single-celled organisms might not be able to travel a meter without dying if they did not find food or other resources, but a group of cells that cooperates according to these principles to combine into a bird or an insect might be able to travel dozens of kilometers to new food, these principles potentially increase sustainability per unit of resources by orders of magnitude so that processes (in this case life) are reliably self-sustaining. Abstracting these principles is intended to enable them to be applied to functional models of processes used by human groups, to potentially achieve the same self-sustainability in those processes.

\section{General Collective Intelligence as a Reliable Driver of Economic Growth and Protection from Global Tipping Points}

Being defined by the FMF as having general problem solving ability, GCI is defined as having the capacity to solve the general problem of maximizing collective well-being, unlike other decisionmaking systems lacking general problem solving ability, which by definition in the FMF do not have the ability to maximize collective well-being. Since impact on outcomes in the FMF (including impact on well-being) is measured in terms of volume of outcomes per unit of input, and since collective outcomes are measured in terms of volume of outcomes per capita, then a GCI is also defined by the FMF as having the capacity to maximize collective well-being per capita per unit of resources where other group decision-making systems without general problem solving ability do not.

The concept of planetary boundaries [6] may be more appropriate than the concept of global tipping points in describing the changes that current non-adaptive decision-making systems are capable of accommodating. In the past, as mentioned by Hughes T. et al, "some global shifts, such as loss of biodiversity from mass extinctions or shifting biomes during an ice age, were quickly reversed on a geological timescale" [6], which may indicate that global tipping points as "the amount of change in large-scale processes that can be accommodated without severely damaging the biosphere", might be very different from the planetary boundaries as the amount of change that allows current decisionmaking systems to maintain or improve human well-being. Without advocating that any planetary boundaries be exceeded, decision-making systems that replicate the adaptive capabilities of living processes aim to achieve similar or the same adaptability as the biosphere so that stability is achievable within global tipping points rather than only within planetary boundaries.

Assuming that global tipping points have a direct relationship with collective well-being within such an adaptive decision-making system, then global tipping points might much more effectively be avoided 
through the maximization of well-being per unit of resources such as system might be capable of, rather than by trying to solve any system of constraints such as the global tipping points or planetary boundaries. For example, rather than the UK targeting a reduction in carbon emissions of 57\% below 1990 levels by 2030, cooperation orchestrated by a GCI within collectively intelligent value chains might reduce consumption of physical resources, and therefore reduce the carbon emissions accompanying that consumption, to the maximum amount that is possible through any possible chains of cooperation. The UK's target reduction in emissions might be modeled as leveraging one or more chains of cooperation to reduce carbon emissions. However representing the UK as a group consisting of $\mathrm{N}$ businesses and other entities, the total set of potential chains of cooperation that are possible through interactions within the entire group will always be larger than the one or more chains of cooperation that can be defined by any subset of decision-makers [1] but that are a subset of the total. If GCI is used to restrict the chains of cooperation selected to those that create a positive reduction in emissions, no carbon emission reduction possible through a subset of these chains of cooperation can match the maximum carbon emission reduction that is possible through all potential chains of cooperation. And solving the general problem of maximizing the use of all chains of cooperation requires the general problem solving ability of a General Collective Intelligence [1].

\section{A Specific Example}

Collective intelligence has the potential to provide powerful competitive advantage for groups of businesses that cooperate to do local collective good. As a conceptual example, consider a fictitious project to improve livelihoods in South Sudan. Assume that NGOs are currently trying to create jobs by running tailor training programmes. But unfortunately, there's no clothing industry to hire the people they train, so this training doesn't reliably create jobs. Virtually all clothing is imported, and though cotton is a big crop, virtually all of it is exported. The value chain is broken, as in many cases elsewhere. But assume that with the uniforms needed for school children each year in South Sudan, there is the potential to create as many as $10,000-12,000$ tailoring jobs if all the uniforms weren't imported. This might be about a thousand-fold increase over the few dozen tailors that are currently trained and that for the most part don't get jobs. To fix this broken value chain, it may be possible to define a chain of cooperating businesses. The first business might distribute school uniforms. To guarantee demand the business might offer stores that sell school uniforms a 50\% discount for the first year in return for signing up to buy those uniforms for the next 5 years. We can then define a business on the to train tailors, a business to manufacture the uniforms and hire the tailors, a business to weave the cloth from local cotton, and a business to source the cotton from local growers for the same 5-year period. Each of these businesses cooperate though agreeing to buy key products or services from another business in the value chain, in return for another business in the chain agreeing to buy from them.

Each of these projects will be profitable, and so can be funded through private investment. An additional opportunity for cooperation comes in finding donors or government programs who are already committed to funding such job creation. Rather than these donors or governments having to pay $100 \%$ of the cost of funding new businesses in order to create jobs, they could be offered the opportunity to cooperate with this program by providing a much smaller amount of funding merely to incentivize private investment. Having only to incentize private investment rather than funding an entire business on their own might allow donors or governments to fund only perhaps $20 \%$ of a programme, thereby multiplying impact per dollar by 5 times.

Furthermore, new businesses are at best only likely to succeed $10 \%$ of the time. So funding new businesses as a way of creating jobs is only likely to be successful $10 \%$ of the time. However using a "pay for success" approach in which the donor or sponsoring government pays that $20 \%$ through 
impact bonds that commit it to reimbursing each project only AFTER it creates the jobs, the probability of project success for the donor might increase from that $10 \%$ to near $100 \%$ per donor dollar. The businesses still won't be successful in creating jobs $100 \%$ of the time, but because the donor or government only pays when they are, that government or donor will be $100 \%$ successful in its job creation per dollar spent. In going from $10 \%$ success in creating jobs to $100 \%$ success per dollar spent, the donor or government will multiply the impact of their funding by a further $10 \mathrm{X}$. The total increase in impact per dollar for the donor in cooperating with this program is then up to 5 times multiplied by 10 times, or 50 times. Because these impact bonds can also potentially be financed in terms of tax credits, and because tax credits on new economic growth don't reduce government income, the growth that can potentially be driven through such impact bonds is limited only by the capacity of the market to absorb impact.

Once successfully piloted, a value chain can potentially be replicated with private investment in a number of other locations. Assume that pilot was replicated in 15 different places. Then in this example of deploying just a few of the many potential patterns of cooperation, the result is a total increase in impact per donor dollar of up to $5 \mathrm{X}$, multiplied by $10 \mathrm{X}$, multiplied by $15 \mathrm{X}$, or $750 \mathrm{X}$. This is a significant multiplication of impact. And it doesn't require any more funding. All it requires is systematically leveraging cooperation.

One aspect of this cooperation is providing competitive advantage so the value chain can succeed. The South Sudan School Uniform value chain relies on the distributor giving a large enough discount to undercut imports. But this $50 \%$ discount is not only too large for other businesses to compete with, it's also too large for the distributor to give and still stay in business. But once we have a chain of businesses that agree to cooperate in that one agrees to buy key products or services from another business in the value chain, in return for another business in the value chain agreeing to buy from it, we can then split that discount into a subsidy of $10 \%$ from each of the five businesses. By creating value chains of cooperating local businesses, we can in this way reliably expand the size of value chain past five businesses to as many as we like, and therefore increase the subsidy they can collectively offer, until we create potentially unbeatable competitive advantage for local businesses.

This cooperation can potentially shift the behavior incentivized in the group from individual competition to group cooperation. Take consumer electronics as an example. Assume that over the last two years a consumer bought at least half a dozen headsets for conference calls, as they kept breaking. Each time the consumer would have had to discard the entire headset even though only one part was broken, because the headset was essentially designed as one piece. If the headset were designed so that the jack was separate, the cord was separate, the speakers were separate, and all the other pieces were separate, and all of those parts were "human-centric" in being replaceable by the user without any specialized tools or expertise, the consumer would have been able to replace just that one part. Rather than lasting for a few months, the headphones instead might last 20 years. This value proposition could provide those headphones with an unbeatable competitive advantage to consumers. But no current business could survive by planning to sell no more than one set of headphones to each customer during the customer's lifetime. Competition may force companies to either compete to drive consumption as unsustainably as possible through planned obsolescence, or be replaced by another entity that will drive unsustainable consumption in their place. Because of this force, companies might be constrained to select the wrong solutions in terms of sustainability, not just in design, but throughout the entire product or service life-cycle.

Specifically, where the value of cooperation is greater than zero, collective intelligence can be used to remove the barriers to scaling cooperation so the value of that cooperation can be increased as much as 
needed to reliably create unbeatable competitive advantage for those businesses that cooperate towards the common good, in this case cooperating to reduce consumption. In the case of the headset, this means creating the capability to search for every opportunity for every business to gain from sharing a design component, making it possible to form entire value chains that cooperate to share designs so they might reuse the same part between them. Through achieving economies of scale in sharing the infrastructure used to manufacture that component, or through achieving any other synergies in the entire product life-cycle, it is potentially possible to create value that essentially subsidizes each specific component ("cross subsidization"). As the number of businesses in the cooperative value chain increases, so does the size of the subsidy it can cooperate to afford. Since the value created through cooperation between any subset of businesses must be smaller than the value that can be created through cooperation between a larger subset of businesses, then the size of the value chain could feasibly be increased to undercut any competition, creating a potentially unbeatable competitive advantage that would help guarantee the sales to support the value chain. With this approach the total cost of the headset might be lowered, and the market share might be raised, to the point where a business could quite comfortably sell only one headset per lifetime.

\section{Extending the Example}

Such patterns of intelligent cooperation can be extended not just to scaling the deployment of products or services, but also to scaling the impact of entire categories of processes. Given that every product or service has an entire life-cycle of processes from research and design, to sales, delivery, and service, and given that every product or service relies on infrastructure with a similar life-cycle of processes, this intelligent cooperation might be deployed in stages, and generalized so it can be reused wherever it applies, so that by defining functional models of those processes it might be possible to drive entire economies this way. The Collective Intelligence based Program to Accelerate Achievement of the Sustainable Development Goals (CIPAA-SDGs) targets exactly this goal.

Phase I of the CIPAA-SDGs program focuses on deployment of existing products and services through cooperative value chains that use Social Impact Bonds as instruments to incentivize cooperation in order to help ensure demand. This demand in turn is used to make the economic development, social impact, environmental impact, and other impacts reliably achievable. Phase I creates outputs (the Social Impact Marketplace platform and related technologies) required for phase II.

Phase II of the CIPAA-SDGs program focuses on development of new products and services with the potential for increased impact on targeted outcomes, using Impact Capacity Bonds as instruments to incentivize cooperation in this development. These products and services are then deployed through cooperative value chains that use Social Impact Bonds to help ensure demand. Phase II creates outputs (various GCI based platforms, products, services, and related technologies) required for phase III.

Phase III of the CIPAA-SDGs program focuses on research of new technologies that can be incorporated into the development of new products and services with the potential for yet further increased impact on targeted outcomes, using Impact Capacity Capability Bonds as instruments to incentivize cooperation in this research. This technology is then used in development that use Impact Capacity Bonds to incentivize cooperation in development of these products and services. These products and services are then deployed through cooperative value chains that use Social Impact Bonds to help ensure demand. Phase III creates outputs (the Collaborative Design Platform) required for collective adaptation of all products and services for green growth and sustainability in phase IV and later phases.

\section{The General Case}


In the school uniform production example provided above, each business in a set of businesses that compete individually outside of such a cooperating value chain, can be represented as achieving one or more outcomes $\mathrm{O}_{\mathrm{j}}$ from the total set of outcomes $\mathbf{O}$ achieved within the set of businesses. Representing the set of outcomes achieved by each business as the vector $\vec{O}$, then each business "i" obtains a total value from each outcome $\mathrm{O}_{\mathrm{j}}$ it achieves. This value is given by the benefits minus costs of that outcome represented as: $V_{i}(\vec{O})$

In a cooperative value chain the value obtained by each business "i" potentially includes a subsidy or other value $\mathrm{V}_{\mathrm{ij}}$ that is provided by each other business " $\mathrm{j}$ " as a result of outcome $\mathrm{O}_{\mathrm{k}}$. The value

$V_{i}^{\prime}\left(\vec{O}^{\prime}\right)$ obtained by the "i"'th business in a cooperative value chain as opposed to the value $V_{i}(\vec{O})$ obtained though competing independently is then:

$$
V_{i}^{\prime}\left(\overrightarrow{O^{\prime}}\right)=\sum_{j} \sum_{k} V_{i}(\vec{O})+V_{j k}^{\prime}\left(\vec{O}^{\prime}\right)
$$

The benefit provided by this cooperation is:

$$
\Delta V_{i}=\sum_{j} \sum_{k} V_{j k}^{\prime}\left(\vec{O}^{\prime}\right)
$$

For there to be some incentive for cooperation for all participants, it must be true that:

$$
\Delta V_{i}>0, \text { for all } i
$$

In addition, to increase the impact of this group or chain of cooperating businesses to the point that impact is reliably achievable, the increase in value for the step that ensures demand for the entire chain must be sufficient to offer the customers of that business a compelling value proposition as opposed to the value proposition offered by other competitors acting alone.

The increase in value that can be offered by business "i" is the subsidy provided by the increase in value $\mathrm{V}_{\mathrm{ij}}$ that cooperation provides to each business "i $\mathrm{i}$ " from each other business " $\mathrm{j}$ ".

$$
\Delta V_{i}=V_{S U B S I D Y}
$$

Representing the value that can be obtained through optimizing individual competitiveness (such as

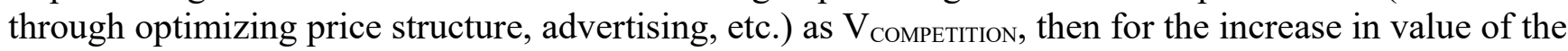
demand ensuring step "i" to be sufficient to offer its customers a compelling value proposition, the subsidy provided by the increase in value to the demand ensuring business that is obtained from cooperation with other businesses must create a stronger value proposition. In other words, it must be true that:

$$
\Delta V_{i}=V_{\text {SUBSIDY }}>V_{\text {COMPETITION }}
$$

By itself this relationship contains little insight. However, assume that in addition to using the South Sudan School Uniform value chain to create tailoring jobs, a second value chain can be constructed to create manufacturing jobs by building modular sewing machines locally. And that a third value chain can be constructed to create software engineering jobs by developing a platform to deliver advertising to all the individuals participating in the value chains in addition to their families. In the general case, a 
value chain might be added if it creates enough value that $V_{\text {SUBSIDY }}>V_{\text {COMPETITION }}$ to ensure sufficient demand for the outcome targeted by the value chain to be reliably achievable.

One way to create sufficient value is to split costs for infrastructure or inputs used by businesses in different value chains so that it is possible to achieve values of scale. Consider the case in which a business incurs a single cost to produce a product. If costs $\mathrm{C}$ are split between $\mathrm{M}$ parties then costs may potentially be reduced by a factor of $\frac{(M-1)}{M}$

Therefore, assuming equal value sharing for simplicity, the value due to economy of scale $\mathrm{V}_{\mathrm{i} \text {, SCALE }}$ that can potentially be obtained by the " $i$ "th business in some number " $M$ " of such parallel cooperating value chains is:

$$
V_{i, S C A L E} \geqslant C_{i} \frac{(M-1)}{M}
$$

The larger the number "M" of cooperating value chains, the larger the value of the potential direct subsidy. In addition, value chains might also include indirect subsidies. Rather than cooperating to reduce a given cost, the group of businesses might just agree to provide the previously mentioned monetary subsidy $\mathrm{V}_{\text {SUBSIDY }}$ to the demand ensuring business in return for the opportunity to participate in the additional sales represented by that additional value chain. So chains of cooperation can potentially engage in chains of cooperation. The larger the system the more chains of cooperation that might potentially be incorporated and the greater the competitive advantage the group can obtain from both economies of scale and subsidies. However, the system comprised by all these chains of cooperation is itself constrained to a maximum size by the business environment. Within such an environment a cooperating value chain might increase the number of cooperators $\mathrm{N}$ in each value chain, and might increase the number of cooperating value chains $\mathrm{M}$ in order to gain competitive advantage. An individual competitor however might increase the geographic regions that it leverages profit from (i.e. globalize) in order to destabilize competition by temporarily undercutting competition in any one region. The total value $\mathrm{V}_{\text {тот }}$ required by an individual competitor to achieve this is:

$$
V_{T O T} \geqslant V_{S U B S I D Y}+V_{i, S C A L E}
$$

A GCI is defined as maximizing collective well-being within the capacity of its processes to do so. So if $\mathrm{M}$ cooperating value chains are selected by a GCI the requirement for each chain of cooperation to be selected is that in addition to the condition above, each chain of cooperation must maximize collective well-being within it's constraints.

[Missing bridge statement]

In the case of businesses competing individually, given $\mathrm{N}$ simultaneous problems $\mathrm{P}_{\mathrm{i}}$, each of which impacts one or more outcomes $\mathrm{O}_{\mathrm{j}}$, then each of these problems might be addressed by a solution $\mathrm{S}_{\mathrm{k}}$. Assuming that each of these problems is a market opportunity that multiple businesses compete to solve, then all of these problems can be solved independently. If each solution that wins a given market share for it's provider is assumed to have done so by maximizing outcomes for a specific customer, then the set of individually competitive solutions that maximizes the total value of outcomes for all customers is represented by $\vec{S}$.

offering a physical or virtual solution $\mathrm{S}_{\mathrm{k}}$ modeled as a sequence of activities, each with 
In the case of businesses cooperating as a group, a functional modeling approach might be used to represent each business " $\mathrm{k}$ " as functioning to consume inputs, and functioning to produce outputs, within some context of execution. This sequence of businesses in cooperative value chain such as the fictional example in South Sudan can be modeled as a pattern of cooperation called an Nth order barter transaction. Representing cooperation as a barter transaction in which one party receives some value in exchange for providing some value in return, then an Nth order sequential interaction to create some product or service can be represented as an Nth order barter transaction in which the first party gives something to the second party, the second party gives something to the third party, and so forth until party $\mathrm{N}-1$ gives something to party $\mathrm{N}$. While opportunities for simple cooperation as represented by first order barter transactions might be easy for human-beings to find and negotiate, complex cooperation as represented by $\mathrm{Nth}$ order barter transactions, as $\mathrm{N}$ becomes large, might be increasingly unmanageable without automation. However, representing complex cooperation in this way as Nth order barter transactions creates the possibility of defining algorithms to find opportunities for such cooperation in design and other processes.

By combining two or more chains of cooperation in parallel, each of which produce an output $\mathrm{O}_{\mathrm{x}}$ from one or more of its businesses, it is possible to increase inputs $\mathrm{I}_{\mathrm{y}}=\mathrm{O}_{\mathrm{x}}$ to an activity which takes that output as its input. This increase in input can be used to scale a specific targeted outcome. Because if step " $x$ " is the rate limiting step in one of many potential processes of cooperation, in general that same outcome $\mathrm{O}_{x}$ might be produced by some step " $\mathrm{y}$ " in the "i”"th process of cooperation. By summing over all process steps in which the outcome is $\mathrm{O}_{\mathrm{x}}$, the scale $\mathrm{M}_{\mathrm{x}}$ to which outcome $\mathrm{O}_{\mathrm{x}}$ can potentially be increased is:

$$
M_{x}=\sum_{i} \sum_{y} \frac{O_{y i}}{O_{x}}
$$

Considering in addition the business processes of each individual business in the value chain, each business offering a physical or virtual solution $S_{k}$ can be modeled as engaging in some sequence of activities. Defining functional models of these activities, chains of cooperation can potentially be defined between business processes as well. Therefore, by defining chains of cooperation representing all possible interactions within a group, and in doing so developing a large enough library of patterns of cooperation, it may then potentially be possible to scale the outcomes of any process to the capacity of the environment. The rate at which patterns of cooperation can be discovered, negotiated, implemented are all dependent on cooperation rather than competition. And for cooperation to be possible each participant y must benefit from participating in that cooperation through the cooperation increasing their outcomes by some factor $C_{y} \geqslant 1$. But, as mentioned, if some chain of cooperation in the set of available chains of cooperation maximizes the benefit of cooperation, then by creating the capacity to maximize the number of chains of cooperation that can be searched, the maximum increase in benefit must be reliably achievable. In this way, the computing power of GCI algorithms can potentially be used to expand cooperation within entire economies until sustainable economic growth is reliably achievable.

\section{Conclusions}

Through solving the problem of providing a common means to measure optimal outcomes, and through solving the problem of providing a stable mechanism to continually adapt to become more fit at their decision-making functions, as well as solving a few other key problems GCI has the potential to make this massive multidimensional optimization of group problems reliably achievable. 
Through these properties, this model of general collective intelligence is designed to find opportunities to create value through cooperation between programs, and is designed to remove the barriers to scaling that cooperation so that this value can be increased to the point that SDGs programs can reliably become essentially self-funding at the scale required to be transformative.

In current decision-making systems parties are constrained to compete to maximize consumption in an unsustainable way, because the barriers to cooperation make other modes of interaction impossible. This results in harm to the collective well-being. Since GCI has the potential to make all group interactions available as chains of cooperation, and since the number of cooperative interactions potentially available within a group of $\mathrm{N}$ participants is $\mathrm{N}$ !, then assuming that individual competitors do not interact, with General Collective Intelligence an exponentially greater number of opportunities to cooperate become available, so that it's possible for the group to reliably gain competitive advantage by cooperating to maximize collective well-being.

\section{References}

[1] Andy E. Williams, General Collective Intelligence and the Constraints to Group Decision-Making, under review

[2] Timothy M. Lenton, Hywel T.P. Williams, On the origin of planetary-scale tipping points, Trends in Ecology \& Evolution, Volume 28, Issue 7, 2013, Pages 380-382, ISSN 0169-5347, https://doi.org/10.1016/j.tree.2013.06.001.

[3] Barry W. Brook, Erle C. Ellis, Michael P. Perring, Anson W. Mackay, Linus Blomqvist, Does the terrestrial biosphere have planetary tipping points?, Trends in Ecology \& Evolution, Volume 28, Issue 7, 2013, Pages 396-401, ISSN 0169-5347, https://doi.org/10.1016/j.tree.2013.01.016.

[4] Reyer, C.P.O., Brouwers, N., Rammig, A., Brook, B.W., Epila, J., Grant, R.F., Holmgren, M., Langerwisch, F., Leuzinger, S., Lucht, W., Medlyn, B., Pfeifer, M., Steinkamp, J., Vanderwel, M.C., Verbeeck, H. and Villela, D.M. (2015), Forest resilience and tipping points at different spatio-temporal scales: approaches and challenges. J Ecol, 103: 5-15. doi:10.1111/1365-2745.12337

[5] Andy E. Williams, A Functional Modeling Framework for Representing and Comparing Models of Consciousness or Cognition, under review

[6] Terry P. Hughes, Stephen Carpenter, Johan Rockström, Marten Scheffer, Brian Walker, Multiscale regime shifts and planetary boundaries, Trends in Ecology \& Evolution, Volume 28, Issue 7, 2013, Pages 389-395, ISSN 0169-5347, https://doi.org/10.1016/j.tree.2013.05.019. 